\title{
Investigation of New Intermetallic Bonds and Molecules by Knudsen Effusion Mass Spectrometry Integrated with ab Initio Calculations
}

\author{
A. Ciccioli ${ }^{*}$ and G. Gigli
}

Dipartimento di Chimica, Università degli Studi di Roma "La Sapienza", p.le Aldo Moro 5, I-00185, Roma, Italy

\begin{abstract}
The recent work of the authors' group in the field of KEMS studies of intermetallic gaseous molecules is reviewed. Examples are given of systems such as intragroup 14 diatomic molecules, -tri- and tetratomic species in the SiSn systems, and diatomic species containing Group 11 elements. Special attention is given to species containing heavy metals, where relativistic effects can play an important role in the stabilization or destabilization of the chemical bond. The results of the $a b$ initio investigation of the same species at various levels of theory, up to CCSD(T), are also reported, showing the importance of combining the experimental and theoretical study of intermetallic molecules.
\end{abstract}

Keywords: Knudsen Effusion Mass Spectrometry (KEMS), Intermetallic molecules, Chemical bond, Atomization energy, DFT calculations, Coupled Cluster calculations.

\section{INTRODUCTION}

Since its beginnings, the Knudsen Effusion Mass Spectrometry (KEMS) technique was recognized to be a powerful tool to discover new molecules and to investigate their energetic properties, such as dissociation and atomization energies [1]. In the last 50 years, many previously unknown molecular species were identified by KEMS in the high temperature vapors produced under conditions close to thermodynamic equilibrium, and their atomization energies determined. The well-known Herzberg's compilation of molecular data of diatomic species [2] witnesses the huge amount of KEMS work in this field up to the late seventies, including oxide, hydride, carbide, halide, picnide species. As pointed out by Leo Brewer in his pioneering studies on high temperature chemistry [3], the particular conditions established in heterogenoeus solidvapor or liquid-vapor equilibria at high temperature may favour the formation of unfamiliar species with elements in unusual 'valence states', so enlarging the gamut of molecular species well beyond those commonly observed under ordinary conditions. Although more and more sophisticated spectroscopic techniques became availaible in the last decades, in many cases the KEMS ('thermochemical') values of dissociation energies listed in the Herzberg's book are still the ultimate reference.

In more recent times, the KEMS technique was successfully applied to the study of intermetallic molecules, both diatomic and, to a smaller extent, triatomic [4] (note that in this context, the term 'intermetallic' is used in a broad sense, including also metalloid elements such as silicon, germanium, etc). The investigation of intermetallic chemical

\footnotetext{
*Address correspondence to this author at the Dipartimento di Chimica, Università degli Studi di Roma "La Sapienza", p.le Aldo Moro 5, I-00185, Roma, Italy; Tel: +390649913640;

E-mail: andrea.ciccioli@uniroma1.it
}

combinations is driven by both fundamental and technological reasons. With regard to the latter, the interest for small metal and semiconductor clusters stems from applications such as new catalysts, electronic devices and nanostructured alloy materials. The knowledge of energetic data, beginning from the dissociation energy of the simple diatomic species, is often needed in assessing and modelling the physicochemical properties of these materials and in optimizing the synthesis conditions. From the fundamental side, the molecules containing from 2 up to $4-5$ atoms are the basic building blocks of larger clusters representing a bridge between the molecular and alloy condensed state, with peculiar features such as the interplay between electronic and geometric effects in determining the size-stability relations [5].

An even more fundamental boost to the study of gaseous intermetallic chemistry comes from the interest for the study of the properties of metal-metal bond itself. Somewhat contrarily to the common belief, many chemical bonds potentially formed between pairs of metal elements have been never observed in the simple diatomic molecule and/or in a small "naked" aggregate of metal atoms. In other words, many of the A-B chemical combinations are not experimentally known. The last comprehensive review on this topic, published by Gingerich [4] around 30 years ago, contains data for about 110 diatomic species (plus about 20 tri- and polyatomic species), and relatively few works has been published since then. The unidentified chemical combinations mainly regard heavy elements. Due to the electronic complexity of the atoms involved, the study of metal-metal bonds offer the possibility to investigate phenomena such as d-electron participation and high bond multiplicity. A further interesting feature of molecules containing heavy elements is that bond properties of these species can be strongly affected by relativistic effects [6,7], owing to the high nuclear charges. While the most pronounced effects regard the inner electrons, whose velocity can approach the light speed, also outer electrons 
are directly or indirectly affected, and so bond energies show significant stabilizing or destabilizing effects. Until the late Eighties, the theoretical prediction of the electronic properties of heavy molecules was essentially qualitative. The relativistic quantum chemistry was a rather secondary branch of theoretical chemistry, and even the non relativistic $a b$ initio treatment of these species was strongly limited by the high computational cost and the lack of adequate computational resources and of generally available computer codes. In the last 25 years much progress has been achieved in the development of advanced computational tools and techniques, and a number of approximate methods were introduced, by-passing the full four-component relativistic treatment. However, although in recent years the relativistic quantum chemistry is attaining maturity, even today the achievement of chemical accuracy in the prediction of atomization energies is still a challenging goal.

The aim of our research [8-11] is to contribute to the understanding of "novel" chemical bonds; i.e. chemical bonds which come into action between a combination of chemical elements never observed before in "naked" molecules and clusters. While this basic goal requires the identification of new diatomic species, the investigation of tri- and polyatomic molecules is also of great interest, pointing towards the field of metal clusters. To this end our efforts are directed to produce with rather simple innovative methods effusive molecular beams into which the target molecules are present in measurable amounts. The KEMS technique allows us to access the strengths of the chemical bonds formed, by measuring the dissociation energy of diatomics or the atomization energy of small aggregates. The focus is on those molecules where, as a consequence of the presence of heavy atoms, a significant relativistic contribution to the bond, both stabilizing or destabilizing, is at work. This research, while experimental in its essence, is complemented with a computational effort, which is closely integrated with the experimental studies, as described in the following sections.

\section{EXPERIMENTAL METHODS}

The experiments are carried out by using the two high temperature mass spectrometers available in our laboratory an older Nuclide apparatus, modified by us in the course of the years, with a single focusing $60^{\circ}$ magnetic sector, and an instrument by Patco, with a single focusing $90^{\circ}$ magnetic sector. Vapors effusing from the Knudsen cell are ionized by electron impact, with an electron emission current generally regulated at $1.0 \mathrm{~mA}$. The energy of ionizing electrons can be continuously changed from 0 to $100 \mathrm{eV}$ in order to investigate the ionization efficiency curves (IEC) and to select the optimal ionization conditions. Appearance potentials of the ions can be determined by the IEC analysis with an uncertainty usually in the range $0.1-0.5 \mathrm{eV}$. Whenever possible, different extrapolation methods were used to evaluate the appearance potentials, and an averaged value was selected as the best estimate. Typically, the vanishing current, linear extrapolation, semilog plot, and extrapolated voltage difference methods were employed. In both instruments the detection of ions is made by a secondary electron multiplier. The conversion of ion currents into partial pressures is carried out according to the well- known KEMS equations, where the cross sections of the newly discovered species may be estimated by a number of semiempirical approaches. As a rule, we used the experimental cross sections for atoms (when available) and an empirically adjusted additivity rule for molecules [ $[10$, 12]. The temperature is typically measured by $\mathrm{Pt} / \mathrm{Pt}-\mathrm{Rh}$ or W-Re/W-Re thermocouples or by an optycal pyrometer sighting into a close-to-black-body cavity in the bottom of the cell. Typical temperature intervals covered in our experiments are in the $1300-2300 \mathrm{~K}$ range.

In order to optimize the conditions for the formation of the target species, two different configurations of the molecular source are used, a classic single-cell source and a modified double-cell assembly similar to that employed by Hilpert and Ruthardt [13]. The choice for the best configuration is mainly dictated by the difference in volatility of the constituent metals and by their alloying properties. Indeed, the optimal conditions favouring the formation of the $A B$ species usually requires that the partial pressures of A and B in the cell be as high and as close each other as possible, provided that the molecular effusion regime be maintened, that is total pressure be well below $10^{-3}$ bar. The fulfillment of these conditions depends both on the vapor pressure of the pure metals and on their activity in the vaporizing condensed phase. Proper conditions may be achieved with a single cell source containing an A-B mixture only when the vapor pressures of the elements are not too different or when the activity of the more volatile metal is sufficiently lowered by A-B interactions in the alloy. In the latter case, the choice of a proper initial composition of the A-B sample is critical. Other alloying elements can be also added to obtain the optimal vapor composition. If the above conditions are not met, a double cell assembly is needed, as shown in Fig. (1). The principal Knudsen cell containing the less volatile element (e.g., A) is suspended with tungsten rods within the hot zone where heating is accomplished with a tungsten resistor coil. A second crucible containing the more volatile component $\mathrm{B}$ is placed below the Knudsen cell, connected to it by a home-made tantalum tube. The lower crucible is not heated but by radiation and thermal conduction from the upper cell, so that its temperature is kept lower than the Knudsen cell (typically by $500-800$ degrees at the temperatures of measurements). A flux of $\mathrm{B}$ vapor enters the Knudsen cell where the gaseous $\mathrm{AB}$ or more complex species are possibly formed. In order to attain the best experimental conditions, proper alloys of $\mathrm{B}$ with $\mathrm{A}$ or other elements can be used in both the cells.

It is important to note that when the vapor of pure B is significantly contributed by species other than monoatomic $\mathrm{B}(\mathrm{g})$ (e.g., the dimer $\mathrm{B}_{2}$ ) the composition of the $\mathrm{B}$ vapor so produced in the upper cell is considerably different from that of the pure metal, due to the thermal dissociation of molecular species. This effect can hamper the study of isomolecular exchange reactions involving the $\mathrm{B}$ dimer or oligomers and can make difficult to identify polyatomic molecules such as $\mathrm{AB}_{2}$ or $\mathrm{A}_{2} \mathrm{~B}_{2}$.

In chosing the molecular source configuration to be used and the composition of the sample(s), activity data of A-B solutions or intermetallic compounds can be useful for an evaluation of the expected partial pressures (at least, their initial values). While experimental activity data at such high 

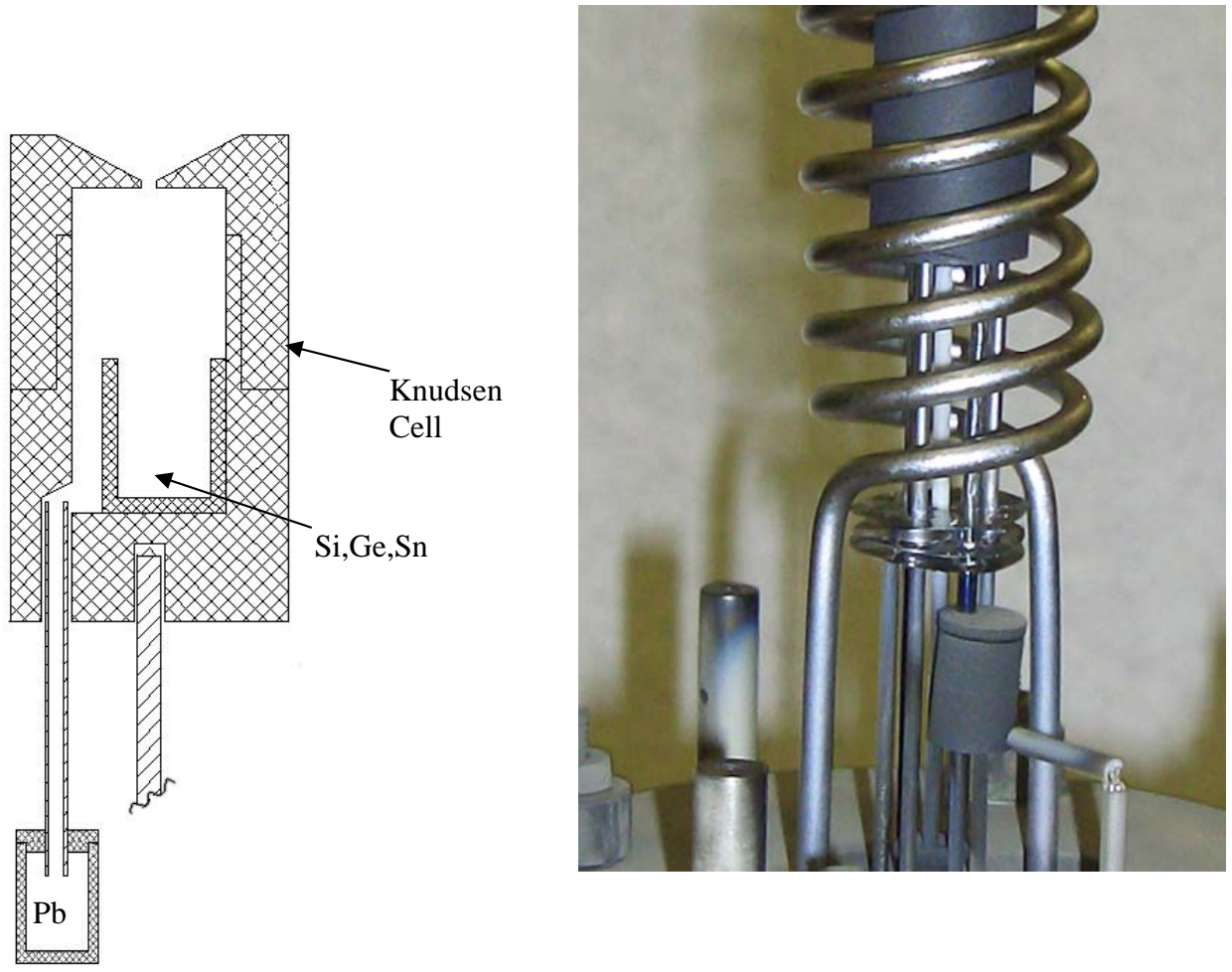

Fig. (1). A simplified scheme (left) of the double cell assembly used in the study of PbM species $(\mathrm{M}=\mathrm{Si}, \mathrm{Ge}, \mathrm{Sn})$, and a practical experimental implementation (right).

temperatures for many binary and most ternary/higher intermetallic systems are often scarce or limited to narrow composition ranges, the thermodynamic information resulting from phase diagram Calphad optimization work [14] can be of help to get a reasonable prediction.

\section{COMPUTATIONAL METHODS}

While the tradition of our research group is basically of experimental type, in the last few years a computational effort was systematically added to our KEMS work. This is due to several reasons: i) our fundamental interest for the study of new chemical bonds with significant relativistic contributions requires a deeper investigation of the nature of these bonds, which can only be achieved by a theoretical analysis; ii) since the fully relativistic treatment of these species has only recently come to maturity, and it remains the domain of very specialized groups and computational tools, it is highly desirable to test the performance of simpler correlated $a b$ initio methods in reproducing the dissociation energies and molecular parameters of the intermetallic species under study; iii) the accuracy of the atomization energies derived by the third-law analysis of KEMS data relies, in addition to the quality of experimental conditions and data, also on the accuracy of the free energy functions of the new species, calculated from molecular parameters such as interatomic distances and angles, harmonic frequencies, anharmonicity constants, energy and degeneracy of electronic terms. In most cases these parameters are not known from experiments, so a theoretical estimate is necessary.

A number of software packages, both commercial and freely available, were developed in the last 20 years, and are continuously updated with implementation of new computational methods and property calculation. Most of our computational work was carried out with the Gaussian 98, Gaussian 03 [15], and NWChem [16] packages.

Among the post-Hartree-Fock, highly correlated, $a b$ initio approaches, we selected the Coupled Cluster with single and double excitations explicitly treated and triple excitations taken into account at a perturbative level, $\operatorname{CCSD}(\mathrm{T})$, as the election method for most calculations. However, due to the huge computational cost of this method, especially if used with high-level basis sets, our computational strategy is usually based on a combination of $\operatorname{CCSD}(\mathrm{T})$ with the by far less time-consuming DFT (Density Functional Theory) approach, applied with the wellestablished B3LYP, BP86, and PW91 functional forms for exchange-correlation. This lighter method is especially apt for the vibrational analysis and, in the case of polyatomic molecules, for a preliminary ample range search for local minima in the potential energy surface.

Owing to the high number of electrons typically contained in the species of our interest, a full electron calculation is often impractical even in the DFT framework. To tackle this problem, the inner electrons of heaviest atoms are usually described by means of effective core potential (ECP), and the calculation is performed on the valence electrons only [17]. Small and large core ECP including scalar relativistic effects (RECP) were published for most elements by the Stuttgart group of Dolg and Stoll $[17,18]$, and the pertinent basis sets for the valence electrons are also available in various publications or on dedicated web sites $[18,19]$. The series of Dunning's type correlation consistent cc-pVnZ basis set [20] were used, with $n$ typically going 


\begin{tabular}{|c|c|c|c|c|c|}
\hline & $\mathrm{C}$ & $\mathrm{Si}$ & $\mathrm{Ge}$ & $\mathrm{Sn}$ & $\mathrm{Pb}$ \\
\hline $\mathrm{C}$ & $\begin{array}{c}\mathrm{C}_{2} \\
607.4 \pm 2.2\end{array}$ & $\begin{array}{c}\mathrm{SiC} \\
445.6 \pm 33.5\end{array}$ & $\begin{array}{c}\mathrm{GeC} \\
455 \pm 11\end{array}$ & $\begin{array}{c}\mathrm{SnC} \\
-\end{array}$ & $\begin{array}{c}\mathrm{PbC} \\
-\end{array}$ \\
\hline $\mathrm{Si}$ & & $\begin{array}{c}\mathrm{Si}_{2} \\
320 \pm 7\end{array}$ & $\begin{array}{c}\mathrm{SiGe} \\
295.4 \pm 8.6\end{array}$ & $\begin{array}{c}\text { SiSn } \\
233.0 \pm 7.8\end{array}$ & $\begin{array}{c}\mathrm{SiPb} \\
165.1 \pm 7.3\end{array}$ \\
\hline $\mathrm{Ge}$ & & & $\begin{array}{c}\mathrm{Ge}_{2} \\
260.7 \pm 6.8\end{array}$ & $\begin{array}{c}\text { GeSn } \\
230 \pm 13\end{array}$ & $\begin{array}{c}\mathrm{GePb} \\
141.6 \pm 6.9\end{array}$ \\
\hline $\mathrm{Sn}$ & & & & $\begin{array}{c}\mathrm{Sn}_{2} \\
183.4 \pm 0.3\end{array}$ & $\begin{array}{c}\mathrm{SnPb} \\
122.6 \pm 4.0\end{array}$ \\
\hline $\mathrm{Pb}$ & & & & & $\begin{array}{c}\mathrm{Pb}_{2} \\
83 \pm 1\end{array}$ \\
\hline
\end{tabular}

Fig. (2). Dissociation energy $(\mathrm{kJ} / \mathrm{mol})$ of intra-group 14 diatomics. Data from ref.s [24] $\left(\mathrm{C}_{2}\right),[25]\left(\mathrm{SiC}_{)},[26]\left(\mathrm{Pb}_{2}\right),[27]\left(\mathrm{GeC}_{)}\right),[11]\left(\mathrm{Si}_{2}\right.\right.$, SiSn, $\left.\mathrm{Sn}_{2}\right)$, [28] (GeSi), [29] ( $\left.\mathrm{Ge}_{2}\right)$, [30] (GeSn), [10] (SiPb, GePb), [9] ( $\left.\mathrm{SnPb}\right)$.

from double to quadruple zeta quality, consistently with the computational cost requested.

As detailed in the following sections for selected cases, the $\operatorname{CCSD}(\mathrm{T})$ values of atomization energies so obtained needs to be refined by extrapolating the results to the complete basis set (CBS) limit to fully include electron correlation [20]. A large part of the residual discrepancy between the theoretical and experimental atomization energies can be attributed to the spin-orbit (SO) contribution $[21,22]$, which is not included in the scalar relativistic pseudopotentials. This effect, due to the interaction of the intrinsic magnetic moments of the electrons with their orbital angular momentum, is particularly strong for heaviest elements and can also lead to split degenerate states. SO interactions often have a significant influence on the dissociation energies, because atomic and molecular effects do not cancel each other. As mentioned in the next sections, different approximate approaches were used to take into account SO effects in the intermetallic species here if interest.

\section{INTRA-GROUP 14 DIATOMIC MOLECULES}

Molecules formed by elements belonging to Group 14 have generated a special interest because of possible applications in areas such as new sensors and cluster materials [23]. However, among the ten intragroup heteronuclaer diatomics, only five (namely, $\mathrm{SiC}, \mathrm{GeC}, \mathrm{SnC}$, $\mathrm{GeSi}$, and $\mathrm{SnGe}$ ) were experimentally characterized until five years ago [9]. To fill this gap, in the last few years we undertook a systematic study of these species, which led us to identify and characterize the new $\mathrm{SnPb}, \mathrm{SiPb}, \mathrm{GePb}$, and SiSn species by KEMS experiments [9-11]. After this work, the knowledge of the dissociation energies of Group 14 diatomics is now almost complete (Fig. 2), with the only exceptions of the $\mathrm{SnC}$ and $\mathrm{PbC}$ species, which remain a challenging goal for future work.

In view of the much higher volatility of lead, the $\mathrm{Pb}$ containing molecules were produced and studied by using a double cell configuration (see Fig. 1), with typical temperature differences of 500-700 degrees between the lower crucible $(\mathrm{Pb}$ reservoir) and the upper Knudsen cell. The following gaseous equilibria were monitored in the overall temperature range $1420-2300 \mathrm{~K}$ :

(a) the direct dissociation reaction $(\mathrm{Me}=\mathrm{Ge}, \mathrm{Si}, \mathrm{Sn})$

$\mathrm{MePb}(\mathrm{g})=\mathrm{Me}(\mathrm{g})+\mathrm{Pb}(\mathrm{g})$ (b) the isomolecular exchange reactions with the Me dimers $(\mathrm{Me}=\mathrm{Ge}, \mathrm{Si}, \mathrm{Sn})$ :

$\mathrm{MePb}(\mathrm{g})+\mathrm{Me}(\mathrm{g})=\mathrm{Me}_{2}(\mathrm{~g})+\mathrm{Pb}(\mathrm{g})$

(c) the isomolecular exchange reactions with the $\mathrm{Pb}$ dimer $(\mathrm{Me}=\mathrm{Si}, \mathrm{Sn})$

$\mathrm{MePb}(\mathrm{g})+\mathrm{Pb}(\mathrm{g})=\mathrm{Me}(\mathrm{g})+\mathrm{Pb}_{2}(\mathrm{~g})$

(d) the isomolecular exchange reactions with the $\mathrm{Me}$ homonuclear dimers and trimers $(\mathrm{Me}=\mathrm{Ge}, \mathrm{Si})$ :

$\mathrm{MePb}(\mathrm{g})+\mathrm{Me}_{2}(\mathrm{~g})=\mathrm{Me}_{3}(\mathrm{~g})+\mathrm{Pb}(\mathrm{g})$

While reaction (a) gives directly the dissociation energy of the diatomic molecule of interest, the analysis of reactions (b)-(d) requires the use of auxiliary atomization energy data for the homonuclear species involved. Note also that the equilibrium constants of reactions (b)-(d) have the advantage of not depending on the value of the sensitivity constant of the instrument, which is known to be a possible source of error in KEMS work. The dissociation energy values resulting from the third-law analysis of the above equilibria resulted in general good agreement and the proposed average values are reported in Fig. (2).

At variance with the $\mathrm{Pb}$-containing molecules, the lower volatility difference between $\mathrm{Si}$ and $\mathrm{Sn}$ permitted to study the Si-Sn system with a single cell molecular source, by vaporizing a $\mathrm{Si}-\mathrm{Sn}$ alloy of proper composition [11]. Under the established experimental conditions, it was possibile in this case to identify a series of polyatomic species beside the simple $\mathrm{SiSn}$ diatomic, namely $\mathrm{Si}_{2} \mathrm{Sn}, \mathrm{SiSn}_{2}, \mathrm{Si}_{3} \mathrm{Sn}, \mathrm{Si}_{2} \mathrm{Sn}_{2}$, and $\mathrm{SiSn}_{3}$ (see the next section). As far as the diatomic species is concerned, gaseous equilibria similar to those listed above for $\mathrm{Pb}$-containing species were exploited to derive the dissociation energy of SiSn, also reported in Fig. (2).

In order to apply the third-law method of analysis, the thermal functions of all the species involved in equilibria (a)(d) are to be evaluated. Since, with few exceptions [31, 32], the molecular parameters of the heteronuclear species of interest were not known, we used the aforementioned computational strategy to calculate the necessary data. For sake of example, a selection of our computational results for the new molecules and for some homonuclear benchmarks is presented in Table 1. As a general remark, we observe that the calculated internuclear distances and harmonic frequencies are in good to acceptable agreement with experimental values even for the most severe benchmarks 
Table 1. Molecular Parameters of Selected Intra-Group 14 Molecules from Experiments and from CCSD(T) Calculations (basis set aug-cc-pVTZ), with RECP for all Atoms Except Si: Electronic State, Term Energy $\left(T_{e}\right)$, Internuclear Distance $\left(r_{e}\right)$, Harmonic Frequency $\left(\omega_{e}\right)$. Data from ref.s [9-11]

\begin{tabular}{|c|c|c|c|c|c|c|c|c|}
\hline & \multicolumn{2}{|c|}{ State* } & \multicolumn{2}{|c|}{$\mathbf{T}_{\mathrm{e}}\left(\mathrm{cm}^{-1}\right)^{*}$} & \multicolumn{2}{|c|}{$\mathbf{r}_{\mathrm{e}}(\hat{A})$} & \multicolumn{2}{|c|}{$\omega_{\mathrm{e}}\left(\mathrm{cm}^{-1}\right)$} \\
\hline & Calculated & Exper. & Calculated & Exper. & Calculated & Exper. & Calculated & Exper. \\
\hline SiSn & ${ }^{3} \Sigma$ & ${ }^{3} \Sigma$ & $\begin{array}{c}0 \\
949\end{array}$ & $\begin{array}{c}0 \\
1629^{* *}\end{array}$ & $\begin{array}{l}2.517 \\
2.406\end{array}$ & - & $\begin{array}{l}350 \\
383\end{array}$ & 360 \\
\hline $\mathrm{GePb}$ & ${ }^{3} \Sigma$ & - & $\begin{array}{c}0 \\
1085\end{array}$ & - & $\begin{array}{l}2.630 \\
2.513\end{array}$ & - & $\begin{array}{l}211 \\
230\end{array}$ & - \\
\hline $\mathrm{SiPb}$ & ${ }^{3} \Sigma$ & - & $\begin{array}{c}0 \\
1036\end{array}$ & - & $\begin{array}{l}2.582 \\
2.465\end{array}$ & - & $\begin{array}{l}321 \\
348\end{array}$ & - \\
\hline $\mathrm{SnPb}$ & ${ }^{3} \Sigma$ & $\begin{array}{l}0^{+}, 1 \\
2,1\end{array}$ & $\begin{array}{c}0 \\
971\end{array}$ & $\begin{array}{c}0,1363[909] \\
2347,5944\end{array}$ & $2.804 * * *$ & - & 167 & 148 \\
\hline $\mathrm{Pb}_{2}$ & ${ }^{3} \Sigma$ & $\begin{array}{l}0_{\mathrm{g}}, 1_{\mathrm{g}} \\
2_{\mathrm{u}}, 1_{\mathrm{u}}\end{array}$ & $\begin{array}{c}0 \\
1168\end{array}$ & $\begin{array}{c}0,5305[3537] \\
5826,7818\end{array}$ & 2.865 & 2.9271 & 139 & 110 \\
\hline $\mathrm{Ge}_{2}$ & $\begin{array}{l}{ }^{3} \Sigma \\
{ }^{3} \Pi\end{array}$ & $\begin{array}{c}0_{\mathrm{g}}, 1_{\mathrm{g}} \\
2_{\mathrm{u}}, 1_{\mathrm{u}}, 0^{+}{ }_{\mathrm{u}}^{+} 0_{\mathrm{u}}^{-}\end{array}$ & $\begin{array}{c}0 \\
416\end{array}$ & $\begin{array}{c}0,114[76] \\
337,711,1193, \\
1305[766]\end{array}$ & 2.374 & 2.368 & 294 & $\begin{array}{c}287.9 \\
286\end{array}$ \\
\hline
\end{tabular}

*All calculations are carried out within the $\Lambda$-S coupling scheme, whereas experimental states refer to spin-orbit splitted levels. Numbers in square brackets are the $\Omega$-averaged $\omega$ - $\omega$ states.

**Attributed to the ${ }^{3} \Pi \leftarrow{ }^{3} \Sigma$ transition [31].

***The difference between internuclear distances of the neutral $\mathrm{SnPb}$ and the negatively charged ion $\mathrm{SnPb}^{-}$is experimentally known as $0.085 \AA$, to be compared with a calculated value of $0.082 \AA$.

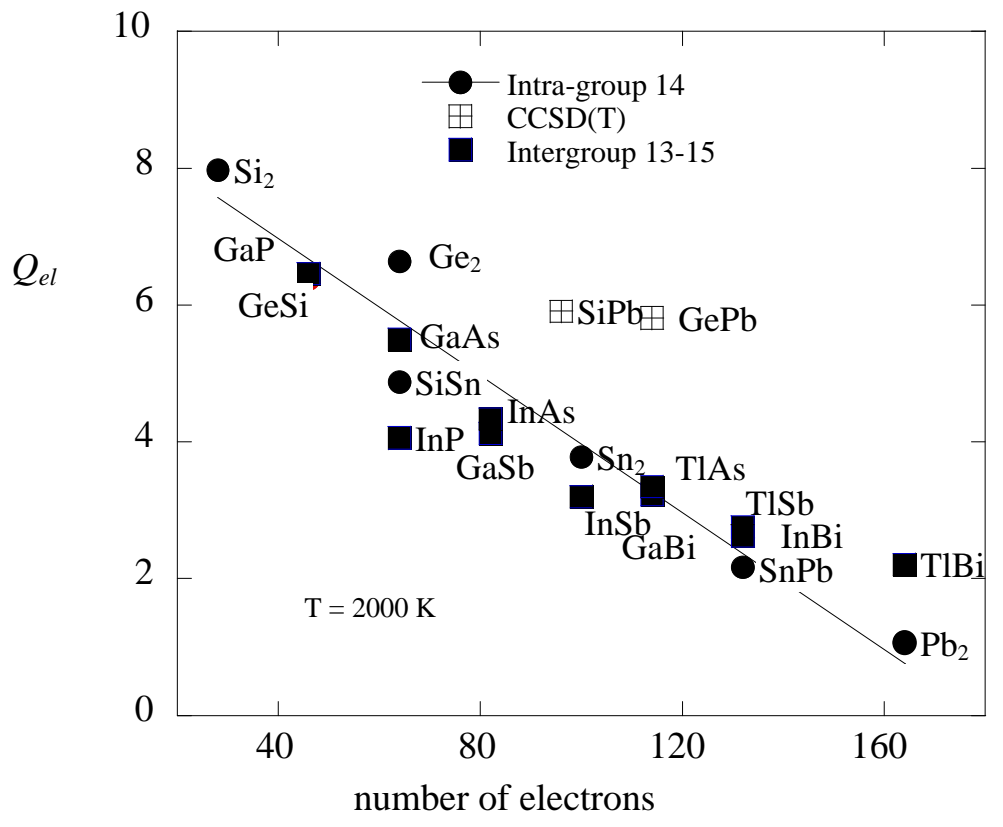

Fig. (3). The electronic partition function at $2000 \mathrm{~K}$ as a function of the number of electrons for intra-group 14 (circles) and intergroup $13-15$ (squares) molecules. It is seen that the values obtained with the scalar RECP - CCSD(T) method for GePb and SiPb deviates markedly from the general trend. Data from ref. [10].

such as $\mathrm{SnPb}$ and $\mathrm{Pb}_{2}$, so that the computed parameters are likely to allow for a reliable computation of rotational and vibrational contributions to the thermal functions.

Quite a different picture occurs if we consider the calculated term energies. This may be expected because the spin-orbit interaction plays an ever increasing role on going down group 14. As a consequence, a pure $\Lambda$-S scheme is no longer valid, the only good quantum numbers being $J$ and $\Omega$, and all the predicted $\Lambda$-S ${ }^{3} \Sigma$ (ground state) and ${ }^{3} \Pi$ (first excited) states are spin-orbit splitted (see Table 1). Let us consider the $\mathrm{Ge}_{2}, \mathrm{SnPb}$, and $\mathrm{Pb}_{2}$ molecules. Using theoretical values would severely overestimate the electronic contribution $\left(Q_{e l}\right)$ to partition function and free energy functions. For example, at $2000 \mathrm{~K}$ the values of $16.70,14.87$, and $14.31 \mathrm{~J} / \mathrm{K}$ mol would be obtained, whereas using the experimentally known term energies would result in 15.74, 6.43 , and $0.66 \mathrm{~J} / \mathrm{K}$ mol contribution, respectively. It is evident that on moving towards heaviest molecules, the spinorbit splitting spreads the levels on an ever increasing energy range. To tackle the problem of the evaluation of the electronic partition function, we exploited an empirical relationship displayed in Fig. (3). The electronic partition function is reported as a function of the number of electrons 
Table 2. Experimental and Theoretical Dissociation Energies ( $\mathrm{kJ} / \mathrm{mol})$ for Intragroup 14 Diatomics. In columns 3 to 5 the Coupled Cluster Theoretical Results Obtained by using Triple Zeta Quality Basis sets, Complete Basis Set (CBS) Limit Extrapolation, and Empirical spin-orbit Correction (see text) are Reported, Respectively. Data from ref.s [10, 11]

\begin{tabular}{|c|c|c|c|c|}
\hline & Experimental (KEMS) & aug-cc-pVTZ-pp CCSD(T) & CBS CCSD(T) & SO-corrected CBS CCSD(T) \\
\hline \hline $\mathrm{SiSn}$ & $233.0 \pm 7.8$ & 255.0 & 274.0 & 242.7 \\
\hline $\mathrm{SiPb}$ & $165.1 \pm 7.3$ & 239.6 & 257.9 & 157.7 \\
\hline $\mathrm{GePb}$ & $141.6 \pm 6.9$ & 237.4 & 252.2 & 143.6 \\
\hline $\mathrm{SnPb}$ & $122.6 \pm 4.0$ & 222.5 & 249.0 & 128.4 \\
\hline
\end{tabular}

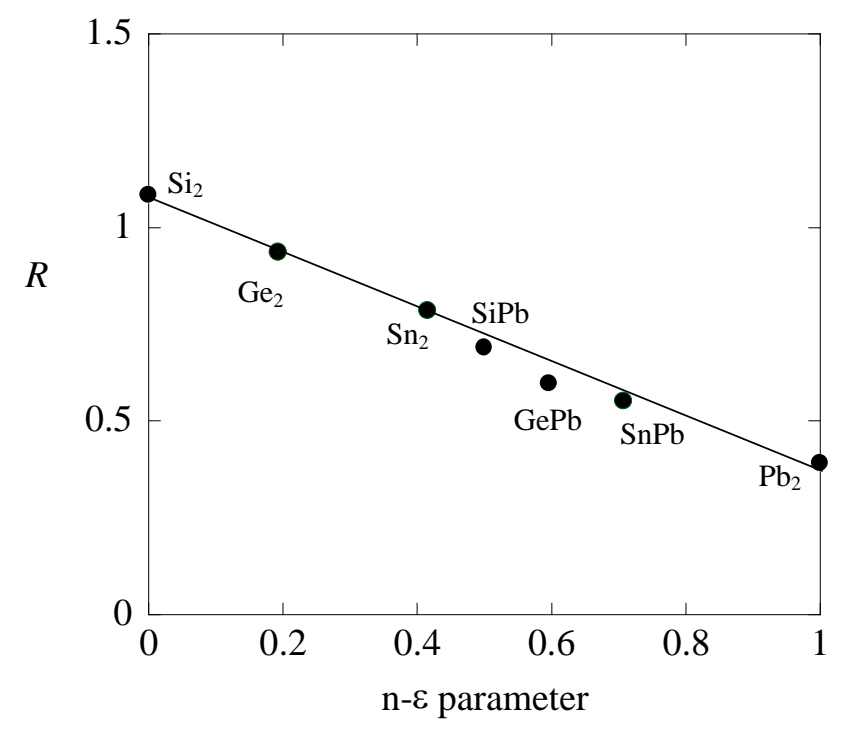

Fig. (4). The ratio $R$ between the experimental and the aug-cc-pVTZ/CCSD(T) theoretical dissociation energies of intra-group 14 molecules $\left(R=D_{0, e x p} / D_{0, \text { theor }}\right)$, reported as a function of the empirical n-e parameter (see text).

for a number of molecules belonging to the intra-group 14 or intergroup 13-15 series, where electronic term energies are available from experiments or from high level relativistic calculations. In this figure, the $Q_{e l}$ estimated using the $\operatorname{CCSD}(\mathrm{T})$ values of the term energies for the $\mathrm{SiPb}$ and $\mathrm{GePb}$ molecules are reported for comparison, showing clearly the overestimate of $Q_{e l}$ which would result. Thus the electronic partition functions for these molecules is better evaluated by using the interpolating line.

With regard to the performance of $\operatorname{CCSD}(\mathrm{T})$ in reproducing the KEMS dissociation energies, similar arguments apply. From the comparison reported in Table 2, it appears that even the CBS-extrapolated values are very far from the experimental results for $\mathrm{Pb}$-containing species, where spin-orbit effects significantly affect the dissociation energy. However, for the lighter $\mathrm{SiSn}$ molecule, the computational value compares well with the experimental result. As detailed in reference [10], a semiempirical correction for the spin-orbit effect both in atoms and in molecules was shown to be effective in reporting the calculated values to a good agreement with experiments (see last column of Table 2). The proposed correction is based on the difference between the lowest spin-orbit splitted level and the spin-orbit averaged state [10,33]. For sake of example, the case of the dissociation energy of the $\mathrm{GePb}$ species is illustrated. For the Ge and $\mathrm{Pb}$ atoms $\left({ }^{3} \mathrm{P}\right.$ term), where accurate spectroscopic data are known, the difference between the lowest $J$ level and the $J$-averaged ${ }^{3} \mathrm{P}$ energy is taken as an estimate of SO correction, giving the values of, respectively, 11.6 and $102.0 \mathrm{~kJ} / \mathrm{mol}$. For the $\mathrm{GePb}$ species, a similar procedure was used, with the $0^{+}-1$ splitting approximately evaluated from the semi-empirical electronic partition function estimated from Fig. (3), giving a value of $4.9 \mathrm{~kJ} / \mathrm{mol}$. Due to the strong SO interaction observed in the $\mathrm{Pb}$ atom, the atomic and molecular effects do not cancel in the dissociation reaction, and the CBS-extrapolated dissociation energy is SO-lowered by almost $110 \mathrm{~kJ} / \mathrm{mol}$, reporting the theoretical value to a very good agreement with the experimental result (see Table 2).

Since the discrepancy between the experimental and the $\operatorname{CCSD}(\mathrm{T})$ (not corrected for spin-orbit effects) dissociation energies displays a clear increasing trend for the heavier species, we managed to find an empirical parameter, so that an as linear as possible trend of the theoretical vs. experimental values difference (or ratio) would be obtained [10] for the series of intragroup 14 diatomics. The following parameter, including both the number of electrons $\left(n_{e l}\right)$ and the amount of spin-orbit effect (as given by the atomic splittings, $\varepsilon_{\text {split,atoms }}$, of the $J$ sublevel of the ${ }^{3} P$ state):

$$
n-\varepsilon=w_{n_{e l}} \frac{n_{e l}-n_{e l_{\min }}}{n_{e l_{\max }}-n_{e l_{\min }}}+w_{\varepsilon_{\text {split }}} \frac{\sum \varepsilon_{\text {split }, \text { atoms }}-\sum \varepsilon_{\text {split atoms }_{\min }}}{\sum \varepsilon_{\text {split atoms }_{\max }}-\sum \varepsilon_{\text {split }, \text { atoms }_{\min }}}
$$



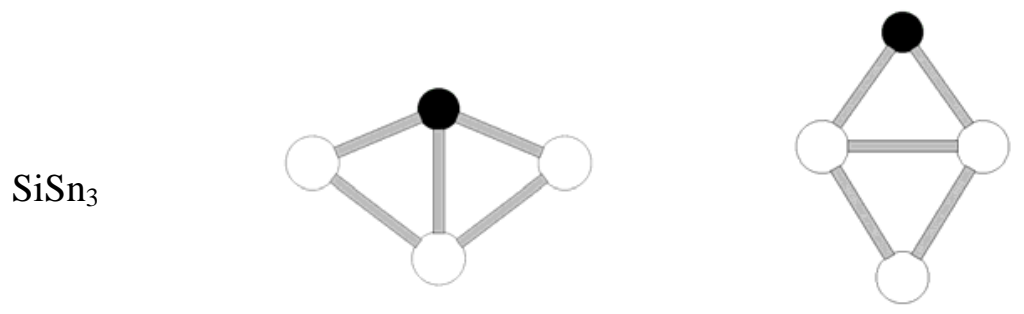

$2585 \mathrm{~cm}^{-1}$

$\mathrm{Si}_{2} \mathrm{Sn}_{2}$
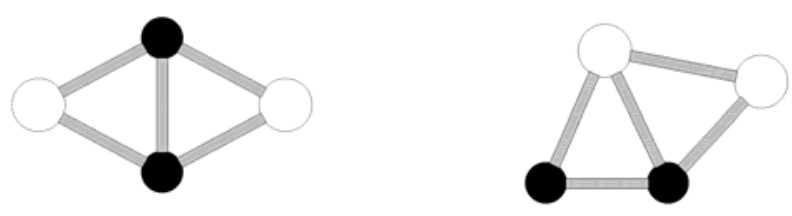

$1150 \mathrm{~cm}^{-1}$
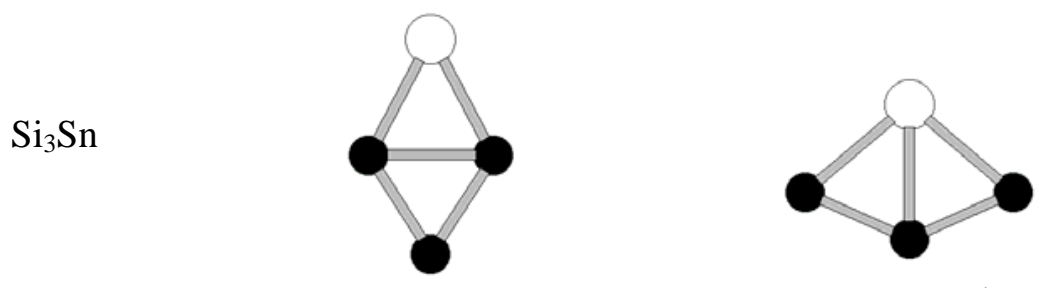

$2518 \mathrm{~cm}^{-1}$

Fig. (5). Geometries of the ground state (left) and the lowest-lying excited structure for the Si-Sn tetratomic species. Numbers below the higher energy structures indicate the energy with respect to the ground state, calculated at the cc-pVTZ-pp CCSD(T) level at the B3LYPDFT optimized geometries. Data from ref. [11].

was found to fulfill at the best this condition, as shown in Fig. (4). In the above equation, $w_{n}$ and $w_{\varepsilon s p l i t}=1-w_{n e}$ are the pertinent weights, to be found by a best fitting procedure, and the subscripts min and max indicate the smallest and the largest values assumed by the two parameters in the series of molecules considered. The so derived trend, together with similar semiempirical procedures, allowed us to derive an overall, reliable estimate of the dissociation energy of the only $\mathrm{Pb}$-containing intra-group 14 diatomic still unidentified - the $\mathrm{PbC}$ species - as $185 \pm 11 \mathrm{~kJ} / \mathrm{mol}$.

\section{INTRA-GROUP 14 POLYATOMIC MOLECULES: THE SI-SN SYSTEM}

As mentioned in the previous section, the experimental conditions used to study the SiSn molecole favoured the formation and identification of several polyatomic species never observed before, namely $\mathrm{Si}_{2} \mathrm{Sn}, \mathrm{SiSn}_{2}, \mathrm{Si}_{2} \mathrm{Sn}_{2}, \mathrm{Si}_{3} \mathrm{Sn}$, and $\mathrm{SiSn}_{3}$. A number of gaseous equilibria involving these species were monitored by measuring the relevant partial pressures with the KEMS technique, including the direct atomization reaction and various isomolecular equilibria with the SiSn species and the homonuclear silicon and tin oligomers. For example, the $\mathrm{Si}_{3} \mathrm{Sn}$ species was studied by exploiting the following equilibria:

$$
\begin{aligned}
& \mathrm{Si}_{3} \operatorname{Sn}(g)=3 \mathrm{Si}(g)+\operatorname{Sn}(g) \\
& \mathrm{Si}_{3} \operatorname{Sn}(g)+2 \operatorname{Si}(g)=\operatorname{SiSn}(g)+2 \mathrm{Si}_{2}(g) \\
& \mathrm{Si}_{3} \mathrm{Sn}(g)+\operatorname{Sn}(g)=\mathrm{Si}_{3}(g)+\mathrm{Sn}_{2}(g)
\end{aligned}
$$

$$
\begin{aligned}
& \operatorname{Si}_{3} \operatorname{Sn}(g)+\operatorname{Si}(g)=\operatorname{SiSn}(g)+\operatorname{Si}_{3}(g) \\
& \operatorname{Si}_{3} \operatorname{Sn}(g)+2 \operatorname{Sn}(g)=3 \operatorname{SiSn}(g)
\end{aligned}
$$

The low number of data collected for each equilibrium suggested to limit the data analysis to the third-law method, and so an as accurate as possible theoretical evaluation of the molecular parameters was necessary to calculate free energy functions of good accuracy.

A rather ample exploration of the potential energy surfaces was carried out for all the species by the B3LYP DFT method, while the higher level CCSD(T) approach was used in selected cases. In particular, energies for the two lowest electronic states were computed for all the polyatomic molecules with the $\operatorname{CCSD}(\mathrm{T})$ method at the B3LYP optimized geometries. The correlation consistent basis sets up to quadruple zeta quality were employed for both silicon, cc-pVXZ, and tin, cc-pVXZ-pp. For Sn, 22 electrons were treated explicitly while a relativistic pseudo-potential accounted for the 28 inner electrons. Finally, the calculated $\operatorname{CCSD}(\mathrm{T})$ atomization energies were extrapolated to the complete basis set (CBS) limit.

For sake of example, in Fig. (5) the ground state and first excited state structures of the tetratomic species are reported. These results can be read in the light of considerations on the relative strength of the $\mathrm{Si}-\mathrm{Si}, \mathrm{Si}-\mathrm{Sn}$, and $\mathrm{Sn}-\mathrm{Sn}$ bonds. Thus, it is evident that the cyclic structure of the $\mathrm{Si}_{3}$ species is basically preserved in the ground state of $\mathrm{Si}_{3} \mathrm{Sn}$, where the $\mathrm{Sn}$ atom is planarly joined to the $\mathrm{Si}_{3}$ framework. The 
Table 3. Experimental and Theoretical Atomization Energies $(\mathrm{kJ} / \mathrm{mol})$ of the $\mathbf{S i}_{\mathrm{x}} \mathrm{Sn}_{\mathbf{y}}$ tri- and Tetratomic Molecules. The Theoretical values are CBS-Extrapolated and Corrected by an Empirical Evaluation of spin-orbit Interaction (see text). Data from ref. [11]

\begin{tabular}{|c|c|c|c|}
\hline & Ground State & Experimental (KEMS) $\boldsymbol{\Delta}_{a t} \mathbf{H}_{\boldsymbol{\theta}}$ & ${\text { Theoretical CCSD(T) } \boldsymbol{\Delta}_{\boldsymbol{a t}} \mathbf{H}_{\boldsymbol{\theta}}}$ \\
\hline \hline $\mathrm{Si}_{3}$ & ${ }^{1} \mathrm{~A}_{1} \mathrm{C}_{2 \mathrm{v}}$ & $716.5 \pm 16$ & 607.6 \\
\hline $\mathrm{Si}_{2} \mathrm{Sn}^{1} \mathrm{~A}^{\prime} \mathrm{C}_{\mathrm{s}}$ & $625.6 \pm 11.6$ & 587.1 \\
\hline $\mathrm{SiSn}_{2}$ & ${ }^{1} \mathrm{~A}_{1} \mathrm{C}_{2 \mathrm{v}}$ & $550.2 \pm 10.7$ & 498.5 \\
\hline $\mathrm{Sn}_{3}$ & ${ }^{3} \mathrm{~A}_{1}{ }^{\prime} \mathrm{D}_{3 \mathrm{~h}}$ & $440 \pm 20$ & 1145.8 \\
\hline $\mathrm{Si}_{4}$ & ${ }^{1} \mathrm{~A}_{\mathrm{g}} \mathrm{D}_{2 \mathrm{~h}}$ & $1160 \pm 22$ & 1073.1 \\
\hline $\mathrm{Si}_{3} \mathrm{Sn}$ & ${ }^{1} \mathrm{~A}_{1} \mathrm{C}_{2 \mathrm{v}}$ & $1046.1 \pm 19.9$ & 998.3 \\
\hline $\mathrm{Si}_{2} \mathrm{Sn}_{2}$ & ${ }^{1} \mathrm{~A}_{\mathrm{g}} \mathrm{D}_{2 \mathrm{~h}}$ & $955.2 \pm 26.8$ & 928.6 \\
\hline $\mathrm{SiSn}_{3}$ & ${ }^{1} \mathrm{~A}_{1} \mathrm{C}_{2 \mathrm{v}}$ & $860.2 \pm 19.0$ & 859.3 \\
\hline $\mathrm{Sn}_{4}$ & ${ }^{1} \mathrm{~A}_{\mathrm{g}} \mathrm{D}_{2 \mathrm{~h}}$ & $750.2 \pm 14.0$ & \\
\hline
\end{tabular}

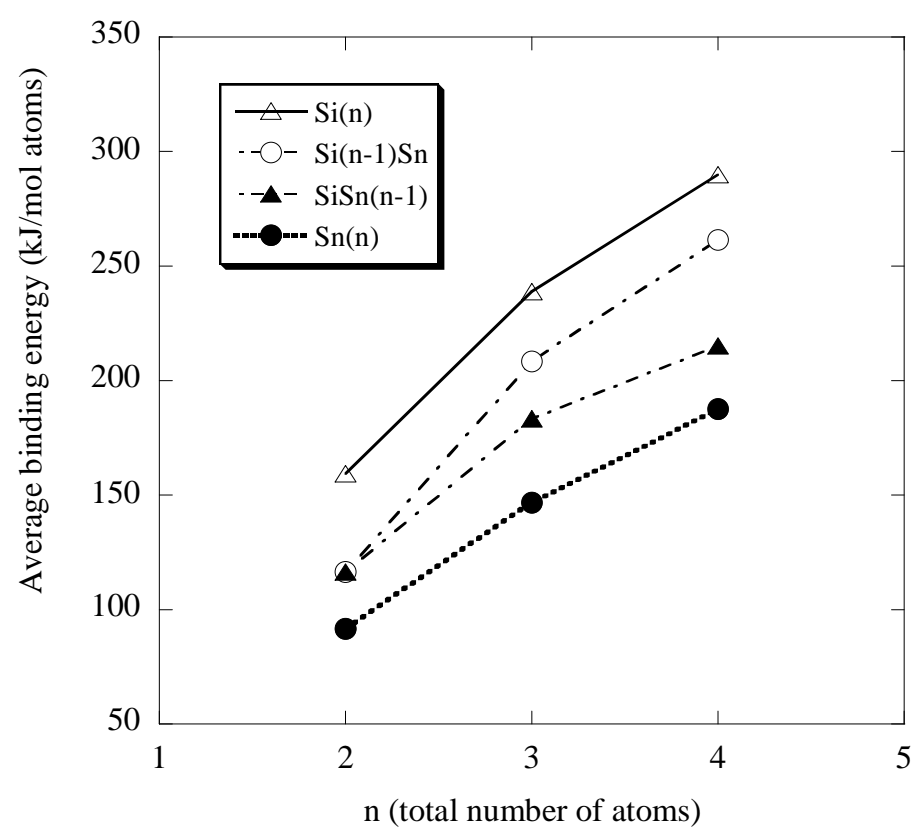

Fig. (6). Trend of the average binding energies of the $\mathrm{Si}_{x} \mathrm{Sn}_{y}$ species as a function of cluster size. Data from ref. [11]

analogous situation is not favoured in the $\mathrm{Sn}_{3} \mathrm{Si}$ species, where the formation of three Si-Sn bonds is preferred, and a fan-like geometry is found in the ground state. The isomer where the $\mathrm{Sn}_{3}$ framework is maintained is found at an energy higher by $2585 \mathrm{~cm}^{-1}$ compared to the ground state. The maximization of Si-Sn bonds is also the driving force for the stabilization of the $D_{2 \mathrm{~h}}$ geometry as the ground state of the $\mathrm{Si}_{2} \mathrm{Sn}_{2}$ molecule (see Fig. 5).

With regard to the comparison between experimental and calculated atomization energies, we report in Table $\mathbf{3}$ the pertinent data for all the studied species. The theoretical results here reported are CBS-extrapolated CCSD $(\mathrm{T})$ single point calculations performed at the cc-pVTZ(-pp) B3LYPDFT optimized geometries. The spin-orbit effect was taken into account by the empirical approach mentioned in the previous section. It is apparent from these data that the deviation between theoretical and experimental results increases progressively on going from $\mathrm{Si}$-rich to $\mathrm{Sn}$-rich clusters. The overestimation of the atomization energies of the heaviest species is most probably due to the approximate treatment of the relativistic effects on the bond.
The study of small polyatomic intermetallic molecules allows one to attempt an analysis of the size-dependence of energetic properties. On this respect, the KEMS technique continues to play a central role in the study of microclusters. Indeed, although a number of more recent techniques became available enabling the production of larger clusters in a wide range of sizes, the energetic study of these species under thermodynamic equilibrium conditions remains an almost exclusive prerogative of KEMS. Among the energetic properties, the average binding energy $E_{b}$, the fragmentation energy $D(n, n-1)$, and the mixing energy $\Delta E_{m i x}$ are of primary interest. They can be evaluated from the experimental atomization enthalpies, as a function of the cluster size by means of expressions such as:

$$
\begin{aligned}
& E_{b}(n)=\Delta_{\text {atom }} \mathrm{H}_{0}^{\circ}\left(\mathrm{SiSn}_{n-1}\right) / n \\
& E_{b}(n)=\Delta_{\text {atom }} \mathrm{H}_{0}^{\circ}\left(\mathrm{Si}_{n-1} \mathrm{Sn}\right) / n \\
& D(n, n-1)=\Delta_{\text {atom }} H_{0}^{\circ}\left(\mathrm{Si}_{n} \mathrm{Sn}\right)-\Delta_{\text {atom }} H_{0}^{\circ}\left(\mathrm{Si}_{n-1} \mathrm{Sn}\right) \\
& \Delta E_{\text {mix }}=-\left[\Delta_{\text {atom }}\left(\mathrm{Si}_{n} \mathrm{Sn}_{m}\right)-\Delta_{\text {atom }}\left(\mathrm{Si}_{n} \mathrm{Si}_{m}\right) n /(n+m)-\Delta_{\text {atom }}\left(\mathrm{Sn}_{n} \mathrm{Sn}_{m}\right) m /(n+m)\right] /(n+m)
\end{aligned}
$$


Table 4. Dissociation Energy (kJ/mol) of Au - Group 2 Metal Diatomics Calculated by DFT (PW91 and BP86 Functionals; cc-pVTZ Basis Set) and Measured by KEMS. Data from ref. [8]

\begin{tabular}{|c|c|c|c|}
\hline & DFT-PW91 & DFT-BP86 & Experimental (KEMS) \\
\hline \hline $\mathrm{AuBe}$ & 246.7 & 239.4 & $234.0 \pm 4.0$ \\
\hline $\mathrm{AuMg}$ & 178.0 & 172.4 & $174.4 \pm 2.7$ \\
\hline $\mathrm{AuCa}$ & 261.1 & 255.6 & $246.7 \pm 4.0$ \\
\hline
\end{tabular}

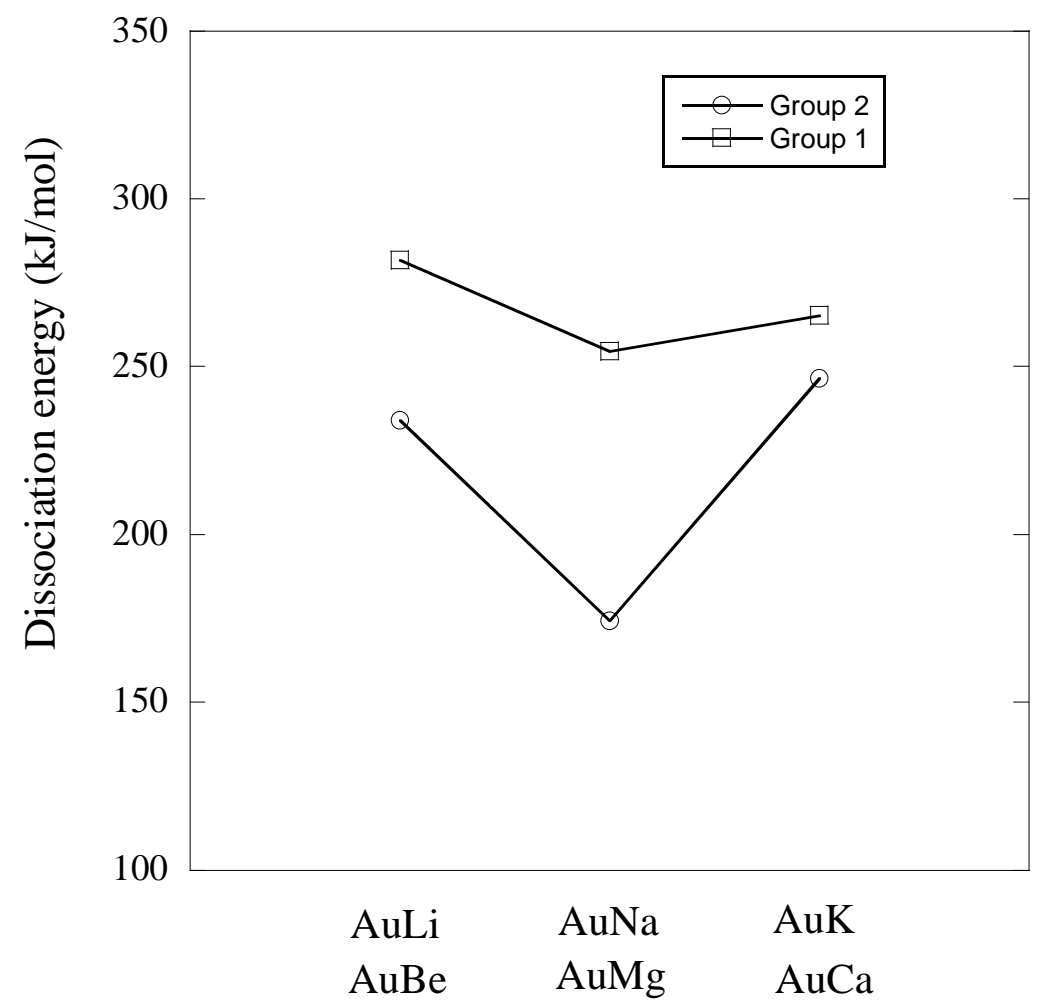

Fig. (7). The V-shaped trend of dissociation energy (experimental values) of group 2 - Au (ref. [8]) and group 1 - Au (ref. [39]) diatomic molecules.

In the case of Si-Sn clusters, we found that the doping of silicon homonuclear clusters with tin has a destabilizing effect, whereas Si-doped Sn clusters are more stable than their homonuclear counterparts (Fig. 6). In the overall, the per atom binding energy shows a monotonic increasing trend, which corresponds to an increasing energetic gain accompanying the growth process. A similar analysis of the fragmentation energies showed that the $\mathrm{SiSn}_{2}$ species is the most stable with respect to the loss of a Sn atom [11].

\section{MOLECULES CONTAINING GROUP 11 ELEMENTS}

Elements belonging to the group 11 of the periodic table (the 'coinage metals' $\mathrm{Cu}, \mathrm{Ag}, \mathrm{Au}$ ) form a very interesting series with regard to the occurrence of relativistic effects. Because of their electronic structure $\left(n s^{1}\right)$, relativistic effects that come into play in the heaviest element are particularly evident, mainly due to the strong relativistic contraction of the $6 \mathrm{~s}$ orbital in gold $[34,35]$.

In order to systematically explore the intermetallic chemistry of gold and other group 11 metals (M), we undertook the KEMS study of selected diatomic molecules, starting with the M-M' systems where $\mathrm{M}^{\prime}$ is a metal belonging to the group 2 of the periodic table. Our initial efforts led us to determine the dissociation energy of the $\mathrm{AuBe}, \mathrm{AuMg}$, and $\mathrm{AuCa}$ species [8]. Further work is currently in progress to complete the series of AuM' diatomics and to explore the $\mathrm{AgM}^{\prime}$ and $\mathrm{CuM}^{\prime}$ species [36]. Furthermore, we succeeded in identifying by KEMS experiments the corresponding $\mathrm{Au}_{2} \mathrm{M}^{\prime}$ molecules, for which the data analysis and the computational evaluation of molecular parameters is also under way.

According to our partial results on the Au-containing species, it is evident that the use of high level basis sets with small core scalar relativistic pseudopotentials leads to very accurate results for both molecular parameters and dissociation energies, even in the DFT approach (Table 4). In particular, the BP86 functional, with the Becke 1988 exchange + the Perdew 1986 correlation contributions [37, 38], seems to be the most effective in reproducing experimental dissociation energies. Another interesting evidence of both experimental and theoretical results is the 
weakness of the $\mathrm{Au}-\mathrm{Mg}$ bond compared to $\mathrm{Au}-\mathrm{Be}$ and $\mathrm{Au}-$ $\mathrm{Ca}$, with a V-shaped trend of the dissociation energies along the group (Fig. 7). The same trend [39] is found also in the Au-M molecules with $\mathrm{M}=$ alkali metal (although the minimum at $\mathrm{Na}$ is much less pronounced in that case; see again Fig. 7) and in non-metallic diatomic molecules of group 2 elements [2].

In order to investigate the effect of strong spin-orbit interactions, an element that deserves much attention is bismuth - the heaviest element in the periodic table among stable elements. For this reason, and in view of the above considerations on group 11 elements, we recently undertook a combined KEMS and ab initio study of the $\mathrm{CuBi}, \mathrm{AgBi}$, and AuBi species. Both single cell and double-cell like configurations were used [40]. In particular, a double cell source was necessary to identify the AuBi species. In order to tackle the computational study of these Bi-containing species, an attempt was done to refine our approach by including a simplified implementation of the spin-orbit effect. To this end, relativistic pseudopotentials were used which take into account spin-orbit interaction with a twocomponent extension [17, 41]. These ECPs were used within the B3LYP-DFT approach, as implemented in the NWChem software package [16].

As a severe benchmark of this approach, a preliminary calculation on the $\mathrm{Bi}_{2}$ species was carried out. The effect of the spin-orbit interaction in this species is suspected to be very significant. Indeed, the CBS-extrapolated $\operatorname{CCSD}(\mathrm{T})$ value of the dissociation energy of this species is calculated at $246.3 \mathrm{~kJ} / \mathrm{mol}$, to be compared with an experimental value of $197.9 \mathrm{~kJ} / \mathrm{mol}$. In order to tentatively correct the highlycorrelated $\operatorname{CCSD}(\mathrm{T})$ result, we performed DFT calculations with both scalar and spin-orbit pseudopotentials. The CBSextrapolated contribution of the spin-orbit correction was evaluated as $-64.9 \mathrm{~kJ} / \mathrm{mol}$, which lowers the $\operatorname{CCSD}(\mathrm{T})$ prediction to $181.4 \mathrm{~kJ} / \mathrm{mol}$, with a considerable improvement in comparison to the uncorrected result. Further calculations are currently in progress on the heteronuclear species which were the subject of the KEMS investigation [42].

\section{CONCLUSION}

In this paper, we presented an overview of the more recent work carried out in our laboratory on the study of intermetallic high temperature molecules by the KEMS techniques, integrated with advanced $a b$ initio calculations.

Our work demonstrates that the KEMS technique can still offer a fundamental contribution to the investigation of new chemical bonds and new intermetallic molecules containing up to a few atoms. With the help of various experimental configurations and conditions, unexplored chemical combinations between many pair of metals may be succesfully investigated.

In particular, the study of heavy species where relativistic effects play an important role in stabilizing or destabilizing the chemical bond permits to obtain precious data to test the performance of high level ab initio calculations such as RECP-CCSD(T), which capture electron correlation energy and include scalar relativistic terms with the use of relativistic pseudopotentials. The analysis of equilibrium data can strongly benefit from theoretical calculations, which provide accurate values of geometrical and vibrational parameters of the ground states, needed for the evaluation of thermal functions. More complete relativistic methods are requested for a rigorous treatment of the spin-orbit effect, which can be nevertheless tentatively taken into account also by proper empirical corrections or by the use of spin-orbit pseudopotentials.

\section{CONFLICT OF INTEREST}

The authors confirm that this article content has no conflicts of interest.

\section{ACKNOWLEDGEMENTS}

Much of the work reviewed in this paper has been contributed by Dr. Giovanni Meloni, to whom we are very indebted. Thanks are also due to dr. Marco Carrozzino, dr. Elena Testani, and dr. Marco Lauricella who collaborated to single papers.

Finally, we are pleased to address our warmest thanks to prof. Giovanni Balducci for his passionate 40-years long work in the KEMS group in Rome.

\section{REFERENCES}

[1] J. Drowart, and P. Goldfinger, "Investigation of Inorganic Systems at High Temperature by Mass Spectrometry," Angewandte Chemie International Edition, vol. 6, pp. 581-596, 1967.

[2] K.P. Huber, G. Herzberg, Constants of diatomic molecules, Van Nostrand Reinhold Company: New York, 1979.

[3] L. Brewer, "Principles of high temperature chemistry," Proceedings of the Robert A. Welch Foundation Conferences on Chemical Research, Nov. 26-28, 1962, VI. Topics in modern inorganic chemistry, chapter III, pp. 47-92.

[4] K.A. Gingerich, "Experimental and predicted stability of diatomic metals and metallic clusters," Faraday Symposia of the Chemical Society, vol. 14, pp 109-125, 1980.

[5] H. Tanaka, S. Neukermans, E. Janssens, R.E. Silverans, and P. Lievens, "Density functional study on structure and stability of bimetallic $\mathrm{Au}_{\mathrm{N}} \mathrm{Zn}(\mathrm{N} \leq 6)$ clusters and their cations," The Journal of Chemical Physics, vol. 119, pp. 7115-7123, 2003.

[6] K. Balasubramanian, Relativistic Effects in Chemistry, Part A and B, Wiley, 1997.

[7] K.G. Dyall, K. Faegri, Introduction to Relativistic Quantum Chemistry, Wiley, 2007

[8] G. Balducci, A. Ciccioli, and G. Gigli, "A mass spectrometric and density functional study of the intermetallic molecole AuBe, AuMg, and AuCa," The Journal of Chemical Physics, vol. 121, pp. 7748-7755, 2004.

[9] G. Gigli, G. Meloni, and M. Carrozzino, "Mass spectrometric and computational study of $\mathrm{SnPb}$ in the gas phase," The Journal of Chemical Physics, vol. 122, pp. 014303-1-6, 2005.

[10] A. Ciccioli, G. Gigli, G. Meloni, E. Testani, "The dissociation Energy of the new diatomic molecole $\mathrm{SiPb}$ and $\mathrm{GePb}$," The Journal of Chemical Physics, vol. 127, pp. 054303-1-16, 2007.

[11] Ciccioli, G. Gigli, and G. Meloni, "The Si-Sn chemical bond: an integrated thermochemical and quantum mechanical study of the SiSn diatomic molecule and small Si-Sn clusters," Chemistry, vol. 15, pp. 9543-9560, 2009.

[12] J. Drowart, C. Chatillon, J. Hastie, and D. Bonnell, "Hightemperature mass spectrometry: instrumental techniques, ionization cross-sections, pressure measurements, and thermodynamic data,' (IUPAC Technical Report) Pure and Applied Chemistry, vol. 77, pp. 683-737, 2005.

[13] K. Hilpert, and K. Ruthardt, Ber. Bunsenges, "Study of the vapor of the chromium-germanium, chromium-tin, and chromium-lead systems: vapor composition and dissociation energy of the intermetallic molecules chromium-germanium ( $\mathrm{CrGe})$, chromiumgermanium $\left(\mathrm{CrGe}_{2}\right)$, chromium-tin $(\mathrm{CrSn})$, and chromium-lead (CrPb)," Physics and Chemistry, vol. 93, pp. 1070-1078, 1989. 
[14] H. L. Lukas, S.G. Fries, and B. Sundman, Computational Thermodynamics. The Calphad method, Cambridge University Press: Cambridge, 2007.

[15] M.J. Frisch, G.W. Trucks, H.B. Schlegel, G. E. Scuseria, M. A. Robb, J. R. Cheeseman, J. A. Montgomery, Jr., T. Vreven, K. N. Kudin, J. C. Burant, J. M. Millam, S. S. Iyengar, J. Tomasi, V. Barone, B. Mennucci, M. Cossi, G. Scalmani, N. Rega, G. A. Petersson, H. Nakatsuji, M. Hada, M. Ehara, K. Toyota, R. Fukuda, J. Hasegawa, M. Ishida, T. Nakajima, Y. Honda, O. Kitao, H. Nakai, M. Klene, X. Li, J. E. Knox, H. P. Hratchian, J. B. Cross, V. Bakken, C. Adamo, J. Jaramillo, R. Gomperts, R. E. Stratmann, O. Yazyev, A. J. Austin, R. Cammi, C. Pomelli, J. W. Ochterski, P. Y. Ayala, K. Morokuma, G. A. Voth, P. Salvador, J. J. Dannenberg, V. G. Zakrzewski, S. Dapprich, A. D. Daniels, M. C. Strain, O. Farkas, D. K. Malick, A. D. Rabuck, K. Raghavachari, J. B. Foresman, J. V. Ortiz, Q. Cui, A. G. Baboul, S. Clifford, J. Cioslowski, B. B. Stefanov, G. Liu, A. Liashenko, P. Piskorz, I. Komaromi, R. L. Martin, D. J. Fox, T. Keith, M. A. Al-Laham, C. Y. Peng, A. Nanayakkara, M. Challacombe, P. M. W. Gill, B. Johnson, W. Chen, M. W. Wong, C. Gonzalez, and J. A. Pople, Gaussian 03 package, Gaussian Inc., Wallingford, CT, 2004.

[16] E.J. Bylaska, W.A. de Jong, N. Govind, K. Kowalski, T.P. Straatsma, M. Valiev, D. Wang, E. Aprã , T.L. Windus, J. Hammond, J. Autschbach, P. Nichols, S. Hirata, M.T. Hackler, Y. Zhao, P.-D. Fan, R.J. Harrison, M. Dupuis, D.M.A. Smith, J. Nieplocha, V. Tipparaju, M. Krishnan, A. Vazquez-Mayagoitia, Q. Wu, T.V. Voorhis, A.A. Auer, M. Nooijen, L.D. Crosby, E. Brown, G. Cisneros, G.I. Fann, H. FrÃ?chtl, J. Garza, K. Hirao, R. Kendall, J.A. Nichols, K. Tsemekhman, K. Wolinski, J. Anchell, D. Bernholdt, P. Borowski, T. Clark, D. Clerc, H. Dachsel, M. Deegan, K. Dyall, D. Elwood, E. Glendening, M. Gutowski, A. Hess, J. Jaffe, B. Johnson, J. Ju, R. Kobayashi, R. Kutteh, Z. Lin, R. Littlefield, X. Long, B. Meng, T. Nakajima, S. Niu, L. Pollack, M. Rosing, G. Sandrone, M. Stave, H. Taylor, G. Thomas, J. van Lenthe, A. Wong, Z. Zhang, NWChem, a computational chemistry package for parallel computers, version 5.0, Pacific Northwest National Laboratory: Richland, WA, 2006.

[17] D. Figgen, G. Rauhut, M. Dolg, and H. Stoll, "Energy-consistent pseudopotentials for group 11 and 12 atoms: adjustement to multiconfiguration Dirac-Hartree-Fock data," Chemical Physics, vol. 311, pp. 227-244, 2005

[18] Available from: http://www.theochem.uni-stuttgart.de/pseudopotentials/index.en.html

[19] Available from: https://bse.pnl.gov/bse/portal

[20] K.A Peterson, D.E. Woon, and T.H. Dunning, Jr., "Benchmark calculations with correlated molecular wave functions. IV. The classical barrier height of the $\mathrm{H}+\mathrm{H}_{2}=\mathrm{H}_{2}+\mathrm{H}$ reaction," The Journal of Chemical Physics, vol. 100, pp. 7410 - 7415, 1994.

[21] D.G. Fedorov, S. Koseki, M.W. Schmidt, M.S. Gordon, "Spin-orbit coupling in molecules: chemistry beyond the adiabatic approximation," International Reviews in Physical Chemistry, vol. 22, pp. 551-592, 2003.

[22] M. Marian, "Spin-orbit coupling in molecules," Reviews in Computational Chemistry, vol. 17, pp. 99-204, 2001.

[23] R. Roucka, J. Tolle, C. Cook, A.V.G. Chizmeshya, J. Kouvetakis, V. D'Costa, J. Menendez, Z.D. Chen, and S. Zollner, "Versatile buffer layer architectures based on $\mathrm{Ge}_{1-\mathrm{x}} \mathrm{Sn}_{\mathrm{x}}$ alloys," Applied Physics Letters, vol. 86, pp. 191912-1-3, 2005.

[24] G. Meloni, L.M. Thomson, and K.A. Gingerich, "Structure and thermodynamic stability of the $\mathrm{OsC}$ and $\mathrm{OsC}_{2}$ molecules by theoretical calculations and by Knudsen cell mass spectrometry," The Journal of Chemical Physics, vol. 115, pp. 4496-4500, 2001.
[25] J. Deng, K. Su, Y. Zeng, X. Wang, Q. Zeng, L. Cheng, Y. Xu, and L. Zhang, "Investigation of gaseous $\mathrm{SiC}(\mathrm{X} 3 \mathrm{P}$ and a $1 \mathrm{~S})$ with accurate model chemistry calculations," Physica A: Statistical Mechanics and its Applications, vol. 387, pp.5440-5456, 2008.

[26] K. S. Pitzer, "Dissociation energies of molecules with very heavy atoms from mass spectrometry," The Journal of Chemical Physics, vol. 74, pp. 3078-3079, 1981.

[27] Levent Sari, Yukio Yamaguchi, and Henry F. Schaefer, “ ${ }^{3} \Sigma^{-}$and ${ }^{3} \Pi$ states of $\mathrm{GeC}$ and $\mathrm{GeSi}$ : The problematic dissociation energy of GeC," The Journal of Chemical Physics, vol. 119, pp. 8266-8275, 2003.

[28] P. Wielgus, S. Roszak, D. Majumdar, J. Saloni, J. Leszczynski, "Theoretical studies on the bonding and thermodynamic properties of $\mathrm{Ge}_{\mathrm{n}} \mathrm{Si}_{\mathrm{m}}(\mathrm{m}+\mathrm{n}=5)$ clusters: The precursors of germanium/silicon nanomaterials," The Journal of Chemical Physics, vol. 128, pp. 144305/1-10, 2008

[29] Shim, M. Sai Baba, and K. Gingerich, "Electronic structure of $\mathrm{Ge}_{2}$ and $\mathrm{Ge}_{2}{ }^{-}$and thermodynamic properties of $\mathrm{Ge}_{2}$ from all electron ab initio investigations and Knudsen effusion mass spectroscopic measurements," Chemical Physics, vol. 277, pp. 9-20, 2002.

[30] R.W. Schmude, Jr., and K.A. Gingerich, "Thermodynamic investigation of small germanium-tin clusters with a mass spectrometer," The Journal of Chemical Physics, vol. 109, pp. 3069-3071, 1998.

[31] S. Li, R.J. Van Zee, W. Weltner, Jr., "Infrared spectra of the GeSi and SnSi molecules in argon matrixes," Chemical Physics Letters, vol. 229, pp. 531-536, 1994.

[32] J. Ho, M.L. Polak, and W.C. Lineberger, "Photoelectron spectroscopy of Group IVA heavy metal dimers: diatomic tin, diatomic lead, and tin-lead mononegative ions $\left(\mathrm{Sn}_{2}^{-}, \mathrm{Pb}_{2}{ }^{-}\right.$, and $\left.\mathrm{SnPb}^{-}\right), "$ The Journal of Chemical Physics, vol. 96, pp. 144-154, 1992.

[33] K.A. Peterson, The Journal of Chemical Physics, vol. 119, pp.11099-11112, 2003.

[34] P. Schwerdtfeger, M. Dolg, W.H.E. Schwarz, G.A. Bowmaker, and P.D.W. Boyd, "Relativistic effects in gold chemistry. I. Diatomic gold compounds," The Journal of Chemical Physics, vol. 91, pp. 1762-1774, 1989.

[35] P. Pyykko and J.P. Desclaux, "Relativity and the periodic system of elements," Accounts of Chemical Research, vol. 12, pp. 276- 281, 1979.

[36] A. Ciccioli, G. Gigli and M. Lauricella, The Journal of Chemical Physics, vol. 136, pp. 184306/1-12, 2012.

[37] A.D. Becke, "Density-functional exchange-energy approximation with correct asymptotic behaviour," Physical Review A, vol. 38, pp. 3098-3100, 1988.

[38] J. P. Perdew, "Density-functional approximation for the correlation energy of the inhomogeneous electron gas," Physical Review B, vol. 33, pp. 8822-8824, 1986 .

[39] G.S.-M. Tong, and A.S.-C. Cheung, "Density Functional Theory Study of Alkali Metal-Noble Metal Diatomic Molecules," The Journal of Physical Chemistry Part A, vol. 106, pp. 11637 - 11643, 2002.

[40] M. Lauricella, "Studio massaspettrometrico e computazionale di molecule intermetalliche contenenti bismuto," Laurea Thesis, University of Rome La Sapienza, 2009,

[41] H. Kim, Y.J. Choi, and Y.S. Lee, "Spin-Orbit and electron correlation effect on the structure of $\mathrm{EF}_{3}(\mathrm{E}=\mathrm{I}$, At, and Element 117)," The Journal of Physical Chemistry Part B, vol. 112, pp. 16021-16029.

[42] M. Lauricella, A. Ciccioli, G. Gigli, manuscript in preparation. 